“ (C) 2018 IEEE. Personal use of this material is permitted. Permission from IEEE must be obtained for all other uses, in any current or future media, including

reprinting/republishing this material for advertising or promotional purposes, creating new collective works, for resale or redistribution to servers or lists, or reuse of any copyrighted component of this work in other works." 


\section{Modelling sustainable supply networks with adaptive agents}

\author{
Subodha Dharmapriya \\ School of Mechanical, Materials, \\ Mechatronic and Biomedical Engineering \\ Faculty of Engineering and Information \\ Sciences \\ University of Wollongong \\ Wollongong, Australia \\ ssd889@uowmail.edu.au
}

\author{
Senevi Kiridena \\ School of Mechanical, Materials, \\ Mechatronic and Biomedical Engineering \\ Faculty of Engineering and Information \\ Sciences \\ University of Wollongong \\ Wollongong, Australia \\ skiriden@uow.edu.au
}

\author{
Nagesh Shukla \\ School of Systems, Management and \\ Leadership \\ Faculty of Engineering and Information \\ Technology \\ University of Technology Sydney \\ Sydney, Australia \\ Nagesh.Shukla@uts.edu.au
}

\begin{abstract}
This paper proposes a multi-agent modelling approach that supports supply network configuration decisions towards sustaining operations excellence in terms of economic, business continuity and environmental performance. Two types of agents are employed, namely, physical agents to represent supply entities and auxiliary agents to deal with supply network configuration decisions. While using the evolutionary algorithm, Non-dominated Sorting Genetic Algorithm-II to optimize both cost and lead time at the supply network level, agents are modelled with an architecture which consists of decision-making, learning and communication modules. The physical agents make decisions considering varying situations to suit specific productmarket profiles thereby generating alternative supply network configurations. These supply network configurations are then evaluated against a set of performance metrics, including the energy consumption of the supply chain processes concerned and the transportation distances between supply entities. Simulation results generated through the application of this approach to a refrigerator production network show that the selected supply network configurations are capable of meeting intended sustainable goals while catering to the respective product-market profiles.
\end{abstract}

Keywords - supply network configuration, adaptive agents, sustainable operations excellence

\section{INTRODUCTION}

Sustainability goals are often discussed in the operations and supply chain management literature in terms of economic, environmental and social dimensions, with some emphasis on the long-term perspective, as well as stakeholder satisfaction [1-4]. However, a growing body of literature claims that pursuit of operations excellence can also contribute to achieving sustainability goals through such means as defects minimisation, waste elimination and stakeholder satisfaction [5-10]. Moreover, in order to sustain operations excellence, organisations must ensure that their operations systems are capable of responding to changes in the business environment, including shifting product-market profiles and competitive dynamics, as well as multiple types of uncertainty [11-14].

In the context of supply networks (SNs), these aspects are dealt with at each stage of value creation and delivery, through appropriate strategic, tactical and operational decisions [14-21]. Considering the relationship between operations excellence and sustainability perspectives highlighted in the literature, this paper explores how this nexus can be reinforced through effective supply network configuration (SNC) decisions. To this end, the paper develops a modelling approach that supports SNC decisions towards sustaining operations excellence in terms of economic, business continuity and environmental performance. As part of this approach, SNs are modelled using adaptive agents to simulate the decision-making behaviour of organisations in a dynamic business setting. The proposed approach helps identify optimal SNCs that satisfy multiple sustainability metrics against a given product-market profile.

The paper is organised into six sections. Following this introduction, a summary of the literature review that informed the study is presented in Section II. The overall modelling approach employed, including a mathematical representation of the SNC problem, is outlined in Section III, followed by an overview of the solution approach proposed in Section IV. The efficacy of the proposed modelling approach is demonstrated using a case study in Section $\mathrm{V}$, and the overall conclusions and future research directions are provided in Section VI.

\section{LITERATURE REVIEW}

The literature on addressing sustainability goals through operational excellence highlights the contribution of the major initiatives such as lean operations, total quality management and reverse logistics [5, 9]. Additionally, pursuing SN level sustainable goals through appropriate governance mechanisms (e.g. supplier assessment, supplier collaboration, multistakeholder initiatives) and leadership roles has been identified as useful initiatives [22-23]. In general, these initiatives aim at improving operations through minimization of waste, efficient use of resources and reuse of material, while meeting the needs of key stakeholders in a rather holistic manner. More recent literature on operations excellence extends this notion by incorporating the need for building organizational capabilities to sustain performance levels attained through above initiatives $[6,12]$. A nuanced perspective on this topic is to consider how sustainable operations excellence can be achieved through appropriate $\mathrm{SN}$ design and operation decisions, or more specifically SNC decisions, at a more fundamental level, which is further explored in this section. 
The range of opportunities and challenges brought about by the expansive global production networks are welldocumented in the literature [15-18]. A globally distributed network of supply entities with distinct capabilities has the potential to create and deliver superior value to end customers, by leveraging the complementary strengths of individual entities and location-specific advantages. At the same time, the complexity of such networks induced by factors such as disparate goals pursued by individual entities and the country or region-specific logistics system attributes may pose significant operational challenges. When these aspects are considered in the context of ongoing changes in the broader business environment such as shifting product-market profiles and competitive dynamics, sustaining operations excellence at the level of SNs can become particularly arduous.

In spite of the above challenges being frequently reported in the literature, efforts directed toward addressing them as part of SNC research has been rather limited. Melo et al. [18] reported that despite the extensive research undertaken on the facilities location problem as part of SN design decisions, it has not been integrated with other SN decisions such as transportation mode choice or routing. While highlighting the importance of considering the aspects such as robustness, resilience and responsiveness when assessing the sustainability of SNs, Klibi et al. [14] claimed that the assumptions used in developing SN models make them too simplistic. Similarly, Eskandarpour et al. [11] called for: broadening the scope of environmental and social measures used in SN design research to include life-cycle approaches; incorporating risk and uncertainty in multi-objective models; and testing SN models in appropriate settings to improve generalizability. Overall, the extant literature highlights the need for extending current SN design research to account for such aspects as uncertainty, dynamic business contexts and multiple performance objectives, as well as following more holistic approaches to the development and testing of SN models [18-19,24-25].

Some recent research efforts have been directed towards addressing the above issues in the context of developing decision support tools. Zhang et al. [20] applied the analytical target cascading method to solve manufacturing service configuration problems involving distributed decision-making. This study demonstrated the effectiveness of the proposed approach in terms of generating the same results as obtained with centralized optimization approaches, while accounting for the autonomous decision objectives of individual SN entities. In a similar vein, Shukla and Kiridena [21] demonstrated the use of a fuzzy rough sets-based multi-agent approach to solve SNC problems, with simulation results confirming the effectiveness of the proposed framework in terms of fulfilling customer orders with lower production and emissions costs, compared to the results generated using comparative existing tools. By comparison Farahani et al. [19] employed a mixedinteger linear programing method, along with simulated annealing and linear relaxation-based heuristics to solve a multi-echelon multi-product $\mathrm{SN}$ involving price-sensitive customer demand zones. The results of this study confirmed the significance of the proposed approach in relation to supporting SNC decisions. Furthermore, a number of review papers published within the current decade have highlighted the contributions made through similar research efforts that have focused on tools supporting SNC decisions to account for sustainability goals in a more encompassing manner $[15,17$, 20-21]. Given the growing interest in the topic of sustainable SN design and the progress witnessed in the area of analytics, there are opportunities for further extending this research towards the development of advanced decision support tools.

\section{PROBLEM REPRESENTATION}

The overall aim of the modelling approach used in this study is to determine the most sustainable SNC that satisfies a given product-market profile, while considering the diverse goals and autonomous decision-making behaviour of individual supply entities involved. Accordingly, the salient features of the modelling environment are: the agent environment; agent attributes and characteristics; and the agent architecture. These features, along with the relevant mathematical notations (refer Table I) relating to the $\mathrm{SN}$ context, are explained below.

\section{A. Agent Environment}

The structural and spatial attributes of the $\mathrm{SN}$ is what represent the agent environment in this paper. We consider a SN with $I$ number of stages $(\mathbf{S})$ where $\mathbf{S}=\left(\mathbf{S}_{I} \ldots \mathbf{S}_{i} \ldots \mathbf{S}_{I}\right)$, which could be any one of the three types, sourcing, manufacturing or distribution. Depending on the bill of material (BOM) of a product, there could be several types of raw material, product component or sub-assembly involved in the each stage. Each of these raw material, component and sub-assembly types is represented as a node in the SN. Accordingly, there could be multiple nodes at any stage. If there is a total of $J$ nodes in the $\mathrm{SN}$, then a node in the $i^{\text {th }}$ stage of the $\mathrm{SN}$ is represented by $\mathbf{N}_{i j}$. At each node, we consider that there are multiple (i.e. $K_{j}$

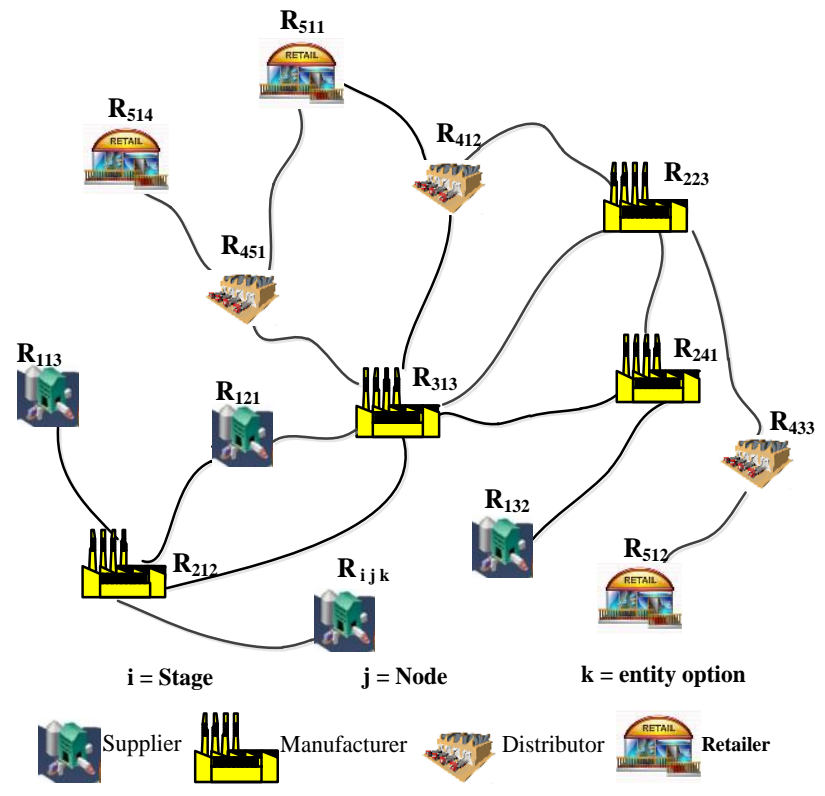

Fig. 1. Representation of a SN 
number of) competing supply entities, termed as entity options $\left(R_{i j k}\right)$, where $R_{i j k} \in \mathbf{N}_{i j}$, they are capable of performing the valueadding functions at the respective node. Depending on such factors as location of facilities, capacity of their plants and the processes or technologies utilized, these entities can compete with each other on cost, lead time or quality parameters. This arrangement reflects the diversity in organizational goals and capabilities observed at the SN entity level. Fig. 1 illustrates this SN graphically with multiple entity options available at each node of a given stage.

\section{B. Agent Characteristics and Architecture}

There are numerous representations of agents (see [26-27] ), agent characteristics (see [26-28] ) and agent architectures (see [29-30] ) reported in the literature. Consistent with the way agents have been defined in the context of SNs, this study treats a SN agent as a human representative or mutually independent task. Agent characteristics are such that they display autonomous (i.e. independently make own decisions) and adaptive (i.e. change the behaviour/decisions upon external influences) behaviour. This behaviour may result from reactive (i.e. respond to the external influences through quick decisions) and pro-active (i.e. take prior initiatives to cope up with future changes) responses, as well as social (i.e. with other supply entities) interactions. Agent architecture is the make-up of an agent in terms of modules and the mechanisms through which these modules interact with each other [31]. Alternatively, agent architecture can be considered as a way of implementing the agent attributes and characteristics [30] introduced earlier. For the purpose of this study the agent architecture consists of three modules, namely, decision-making, learning and communication. The decision-making module is implemented through a rule-based reasoning approach, whereas the learning module is implemented using the $\mathrm{Q}$ learning algorithm. The communication module is used for routing messages.

The decision-making module is designed within an agent to make decisions on $B P_{i j k}$ and $B T_{i j k}$. Within the context of multi agent systems (MAS), Markov decision process (MDP) is used as a mathematical framework for modelling dynamic systems involving sequential decision-making with a defined set of global states, actions, transition probabilities and reward functions [32-33]. MDP can be executed using model-based or model-free algorithms to determine the optimal policy by way of selecting an action in a given state. The optimal decision is awarded with a reward and the next state is determined using a transition probability function. In this study, we use a modelfree algorithm considering the difficulty of developing an accurate model to represent the agent environment due to high variability and dynamic nature. Reinforcement learning is a model-free learning technique, where learning is reinforced through a reward mechanism when interacting with the environment [34]. We implement agent learning process within the learning module by employing what is known as 'temporal difference learning' via the Q-learning algorithm. Q-learning is a model-free learning algorithm which uses the $\mathrm{Q}$ value of an action to predict the best action in the given state in order to maximize a cumulative reward. Once the learning module has updated the Q-table following a successful bidding attempt, the decision-making module selects an action through two methods namely, exploration and exploitation. In exploration, an action is selected randomly hoping that it might give a better reward whereas exploitation trusts the learnt values [32]. This actionselection is done to optimize the defined utility to the agent. The communication module helps to communicate with other connected agents in order to execute both decision-making and learning modules.

\section{Agent Types}

Two types of agents are introduced in this SNC context, namely, physical agents and auxiliary agents. Physical agents are the supply entities namely, supplier (SA) agents, manufacturer (MA) agents and distributor (DA) agents, as they exist in the real world performing typical SN operations. Auxiliary agents are introduced to support SNC decisions by finding optimal SNCs for a given set of product-market profiles. Such auxiliary agents are the order processing (OP) agent, supply entity selection (SES) agents (e.g. supplier selection agent, manufacturing plant selection agent), auctioning (AU) agent, optimizing (OPT) agent, transportation (TA) agent and evaluating (EA) agent. The proposed two types of agents have different architectures. Physical agents consist of all three modules introduced earlier whereas auxiliary agents consist of decision-making and communication modules only.

\section{1) Physical agents}

Physical agents are located in different geographical regions around the world, designated with an identification index (ID) indicating $i, j$, and $k$ respectively. DAs are responsible for storing finished products ready to be dispatched to relevant customers, and MAs for assembling final products. SAs are arranged into a number of tiers according to the BOM of the product. For example, if the SC has three tiers in the supply stage; tier-1 suppliers supply all the sub-assemblies; tier-2 suppliers supply the required parts and/or components; tier-3 suppliers supply raw materials. Those physical agents have their distinct $A C_{i j k}$, and they may periodically increase their production capacity by a percentage of $\gamma$ in a number of different ways (e.g. by adding new machineries or new technology). At the time agents are invited to bid in terms of $B P_{i j k}$ and $B T_{i j k}$, those decisions are facilitated by the decisionmaking and learning modules of the physical agent concerned. These decisions are made based on the parameters such as $R C_{i j k}$ and $A A C_{i j k}$ subject to future expansion strategies.

The common objective of the physical agents is to maximize their $U_{i j k}$ as given in (1). All physical agents follow the same logic in making decisions, with the difference being the value of their attributes such as $P C_{i j k}$ and $P T_{i j k}$. The decision-making process is illustrated in Fig. 2. Block A, B, C, $\mathrm{D}, \mathrm{E}$ and $\mathrm{F}$, highlight how decisions are made in different conditions using learnt knowledge. Learnt knowledge is represented by the Q-table (as given in Table II), in the form of a matrix where rows $(m)$ stand for production capacity status and columns $(n)$ stand for profit ranges. There are five production capacity status $(m)$ as given in (2) to (6). Here, the $A A C_{i j k}$ is the difference between $N C_{i j k}$ and $O C_{i j k}$ as given in (7) where $N C_{i j k}$ is calculated as given in (8) taking $\gamma$ as the percentage increment in production capacity and $O C_{i j k}$ as given in (9), assuming $\mu$ as the percentage of occupied production capacity. $R C_{i j k}$ is equivalent to the number of units required 
TABLE I. MATHEMATICAL NOTATIONS USED IN MAS MODEL

\begin{tabular}{|c|c|}
\hline$i$ & Stages $(i=1,2,3, \ldots, I)$ \\
\hline $\bar{j}$ & Nodes $(j=1,2,3, \ldots, J)$ \\
\hline$k$ & Entity options $\left(k=1,2,3, \ldots, K_{j}\right)$ \\
\hline $\mathbf{S}=\left(\mathbf{S}_{l} \ldots \mathbf{S}_{i} \ldots \mathbf{S}_{I}\right)$ & Set of stages; $\mathbf{S}_{i} \in \mathbf{S}$ \\
\hline $\mathbf{S}_{i}=\left(\mathbf{N}_{i l} \ldots \mathbf{N}_{i j} \ldots \mathbf{N}_{i m}\right)$ & Set of nodes; $\mathbf{N}_{i j} \in \mathbf{S}_{i}$ \\
\hline $\mathbf{N}_{i j}=\left\{R_{i j 1} \ldots R_{i j k} \ldots R_{i j m}\right\}$ & Set of available entity options; $R_{i j k} \in \mathbf{N}_{i j}$ \\
\hline$B P_{i j k}$ & Bidding price \\
\hline$B T_{i j k}$ & Bidding time \\
\hline$U_{i j k}$ & Utility \\
\hline$A C_{i j k}$ & Annual processing capacity \\
\hline$\gamma$ & Percentage capacity expansion rate \\
\hline$R C_{i j k}$ & Required processing capacity \\
\hline$A A C_{i j k}$ & Available annual processing capacity \\
\hline$P C_{i j k}$ & Processing cost \\
\hline$P T_{i j k}$ & Processing time \\
\hline$Q$ & Matrix which represents the Q-table \\
\hline$m$ & Processing capacity status $(m=1,2,3,4,5)$ \\
\hline$n$ & Profit ranges $(n=1,2,3)$ \\
\hline$N C_{i j k}$ & New processing capacity after expansion \\
\hline$O C_{i j k}$ & Occupied processing capacity \\
\hline$\mu$ & Percentage occupied processing capacity \\
\hline$\delta_{i j}$ & $\begin{array}{l}\text { Number of components/subassemblies required } \\
\text { from node } j \text { according to the BOM }\end{array}$ \\
\hline$P_{m n}$ & Profit percentage at given $m$ and $n$ \\
\hline$\beta_{m}$ & $\begin{array}{l}\text { Time coefficient at different } m \\
\left(\beta_{1}=1 ; \beta_{2}=1.15 ; \beta_{3}=1.5 ; \beta_{4}=1.6 ; \beta_{5}=1.75\right)\end{array}$ \\
\hline$V_{l}$ & $\begin{array}{l}\text { Volume of the product-market profile at } \\
\text { consumer region } l\end{array}$ \\
\hline$L T_{l}$ & $\begin{array}{l}\text { Lead time of the product-market profile at } \\
\text { consumer region } l\end{array}$ \\
\hline$P_{l}$ & $\begin{array}{l}\text { Estimated WTP price of the product-market } \\
\text { profile at consumer region } l\end{array}$ \\
\hline$\mu_{3}, \mu_{2,} \mu_{1}$ & $\begin{array}{l}\text { Percentage contribution of profit as reward }\left(\mu_{3}\right. \\
\geq \mu_{2} \geq \mu_{1)}\end{array}$ \\
\hline$R P_{i j}$ & Reserved price for node $\mathbf{N}_{i j}$ \\
\hline$R T_{i j}$ & Reserved time for node $\mathbf{N}_{i j}$ \\
\hline$P P_{i j}$ & Percentage price for node $\mathbf{N}_{i j}$ \\
\hline$P T_{i j}$ & Percentage time for node $\mathbf{N}_{i j}$ \\
\hline$\alpha$ & $\begin{array}{l}\text { Unit distance transportation cost in between } \\
\text { stages }\end{array}$ \\
\hline$D_{i j k \rightarrow i j^{\prime} k^{\prime}}$ & $\begin{array}{l}\text { Distance between two entity options }\left(R_{i j k} \text { and }\right. \\
\left.R_{i^{\prime} j^{\prime} k^{\prime}}\right)\end{array}$ \\
\hline$V$ & $\begin{array}{l}\text { Average speed of the transportation method } \\
\text { used }\end{array}$ \\
\hline$D_{k k^{\prime}}$ & Distance matrix \\
\hline$\overline{y_{i j k}}$ & 1: if entity option is selected; 0 : otherwise \\
\hline$x_{i j k \rightarrow i^{\prime} j^{\prime} k^{\prime}}$ & $\begin{array}{l}\text { 1: if there is a connection between two entity } \\
\text { options; } 0 \text { : otherwise }\end{array}$ \\
\hline$T T_{i j k \rightarrow i^{\prime} j^{\prime} k^{\prime}}$ & Transportation time between two entity options \\
\hline$T C_{j k \rightarrow i^{\prime} j^{\prime} k^{\prime}}$ & Transportation cost between two entity options \\
\hline
\end{tabular}

from each node as given in (10). There are three profit ranges $(n)$ namely, low $\left(5-10 \%\right.$ of the $\left.P C_{i j k}\right)$, medium $(10-15 \%$ of the $\left.P C_{i j k}\right)$, and high $\left(15-20 \%\right.$ of the $\left.P C_{i j k}\right)$. The profit $\left(P_{m n}\right)$ corresponding to the each state-action cell is given in Table II. The value corresponding to each state-action of the Q-table is updated based on the reward that the agent gained through the
Objective of an agent

$\mathrm{Z}=\operatorname{Maximize}\left(U_{i j k}\right)$

Conditions for each production capacity status

$\mathrm{m}=1 \quad A A C_{i j k} \geq 0.5 * N C_{i j k}$ and $A A C_{i j k} \geq R C_{i j k}$

$\mathrm{m}=2 \quad A A C_{i j k} \geq 0.5 * N C_{i j k}$ and $A A C_{i j k}<R C_{i j k}$

$\mathrm{m}=3 \quad A A C_{i j k} \leq 0.5 * N C_{i j k}$ and $A A C_{i j k} \geq R C_{i j k}$

$\mathrm{m}=4 \quad A A C_{i j k} \leq 0.5 * N C_{i j k}$ and $A A C_{i j k}<R C_{i j k}$

$\mathrm{m}=5 \quad A A C_{i j k}=0$

Calculation of available production capacity

$A A C_{i j k}=N C_{i j k}-O C_{i j k}$

Calculation of new production capacity after

$N C_{i j k}=(1+\gamma)^{*} A C_{i j k}$

Calculation of occupied production capacity

$O C_{i j k}=\mu^{*} A C_{i j k}$

Calculation of required production capacity

$R C_{i j k}=\delta_{i j} * V_{l}$

Exploration strategy

$n=$ rand $(1,2,3)$

Calculation of bidding price

$B P_{i j k}=P C_{i j k}\left(1+P_{m n}\right)$

Calculation of bidding time

$B T_{i j k}=\beta_{m} * P T_{i j k}$

Exploitation strategy

$n=\max (Q(m,:))$

Update Q-table with a positive reward

$Q_{m n}=Q_{m n}+\mu_{1}\left(\delta_{i j} * V_{l} * P_{m n}\right)$

Update $\mathbf{Q}$ table with a negative reward

$Q_{m n}=Q_{m n}-\mu_{2}\left(\delta_{i j} * V_{l} * P_{m n}\right)$

Reserved price for node $\mathbf{N}_{\mathbf{i j}}$

$R P_{i j} \sim \operatorname{rnd}\left[P P_{i j} * P_{l}, 0.85 * P P C_{i j} * P_{l}\right]$

Reserved time for node $\mathbf{N}_{\mathbf{i j}}$

$R T_{i j} \sim \operatorname{rnd}\left[P T_{i j} * L T_{l}, 0.85 * P P T_{i j} * L T_{l}\right]$

Bidding price constraint

$B P_{i j k} * y_{i j k} \leq R P_{i j}$

Bidding time constraint

$B T_{j k} * y_{i j k} \leq R T_{i j}$

bidding process. At the very first bidding instance in the case of a new product, the value of each entry is set to zero.

Upon receiving an invitation to bid, the agent checks whether the invitation is for a new product (haven't supplied earlier) or it is one of the regular products supplied. If the invitation is for a new product and is first-time bidding for that product, the agent executes block $\mathrm{A}$. The agent decides on $m$ by calculating and considering the agent properties as per (2) to (6). Then an exploration strategy is adopted to determine $n$ as in (11). Accordingly, $P_{m n}$ is decided referring to Table II and 
TABLE II. REPRESENTATION OF Q-TABLE WITH P $\mathrm{P}_{\text {MN }}$ VALUES

\begin{tabular}{|c|c|c|c|c|}
\hline \multicolumn{2}{|c|}{$\left(\mathrm{P}_{\mathrm{mn}}\right)$} & \multicolumn{3}{c|}{ n } \\
\cline { 3 - 5 } \multicolumn{1}{|c|}{} & $\mathbf{1}$ & $\mathbf{2}$ & $\mathbf{3}$ \\
\hline \multirow{4}{*}{} & $\mathbf{2}$ & $5 \%$ & $10 \%$ & $15 \%$ \\
\cline { 2 - 5 } & $\mathbf{3}$ & $7 \%$ & $11 \%$ & $16 \%$ \\
\cline { 2 - 5 } & $\mathbf{4}$ & $8 \%$ & $12 \%$ & $17 \%$ \\
\cline { 2 - 5 } & $\mathbf{5}$ & $10 \%$ & $15 \%$ & $18 \%$ \\
\hline
\end{tabular}

$B P_{i j k}$ is calculated as given in (12). $B T_{i j k}$ is determined as given in (13) in which $P T_{i j k}$ is multiplied by a defined constant based on the status of the production capacity (i.e. $\beta_{m}$ ). If the invitation is for a first-time bid, but for a regular product (already being supplied), then block B is executed to determine the bidding values. After finding the relevant $m$ according to (2) to (6), the value of $n$ is determined as indicated in (14) through exploitation strategy which is the highest reward in state $m$. Then $B P_{i j k}$ and $B T_{i j k}$ are calculated as given in (12) and (13). In both of the above instances, if the invitation is not for the first time, then the status of the previous bid for the same product-market profile is checked. If the previous bid was won by the agent, as indicated in block $\mathrm{C}$, the Q-table is updated with a positive reward as given in (15) and the next round of bidding is done using a lower $n$ than in the previous bid. Similarly, if the agent had lost the previous bid, as indicated in block $\mathrm{D}$, then the $\mathrm{Q}$ table is updated with a negative reward as given in (16) and the next round of bidding is done using a lower $n$ than in the previous bid. Block $\mathrm{E}$ and $\mathrm{F}$ are executed by checking the capability of the agent to bid with a lower $n$ than in the previous round of bidding. If the agent is capable of finding a lower $n$ than the previous round of bidding, then block $\mathrm{E}$ is executed following an exploration strategy (as in
(11)) to make the bid. If it is not possible to find a lower $n$ than the previous round of bidding, then again upon checking the bidding status (won/lost) in the previous round of bidding, the agent makes a decision to whether to bid with the same value or quit bidding for that order.

\section{2) Auxiliary agents}

Auxiliary agents generate alternative SNCs to suit different product-market profiles. Those auxiliary agents primarily have only decision-making and communication module as they act as executing agents, triggered by a message.

OP agent: calculates the number of units required from the relevant $\mathbf{N}_{i j}$ taking into account both $V_{l}$ of the product-market profile and the BOM as given in (10).

AU agent: determines a set of optimal $R P_{i j}$ and $R T_{i j}$ values seperately using Genetic Algorithm rules for relevant $S N$ nodes to support SES agents to determine which physical agents have been successful in their bidding. This set of optimal reserved values serve two purposes. First, to optimize the SN level performance both in terms of cost and lead time, and the second, to create a degree of competition among physical agents. The initial set of $R P_{i j}$ and $R T_{i j}$ (i.e. initial population) for GA are randomly generated within the defined upper and lower threshold values for each node as given in (17) and (18) respectively. For example, the upper threshold $R P_{i j}$ value is determined taking the $P_{l}$ of the product-market profile and $P P_{i j}$ of the respective node. The initial population is then subjected to genetic operators such as mutation and crossover until termination criteria are met.

Once the AU agent has generated a set of feasible optimal $R P_{i j k}$ and $R T_{i j k}$, it starts auctioning using those values as the first

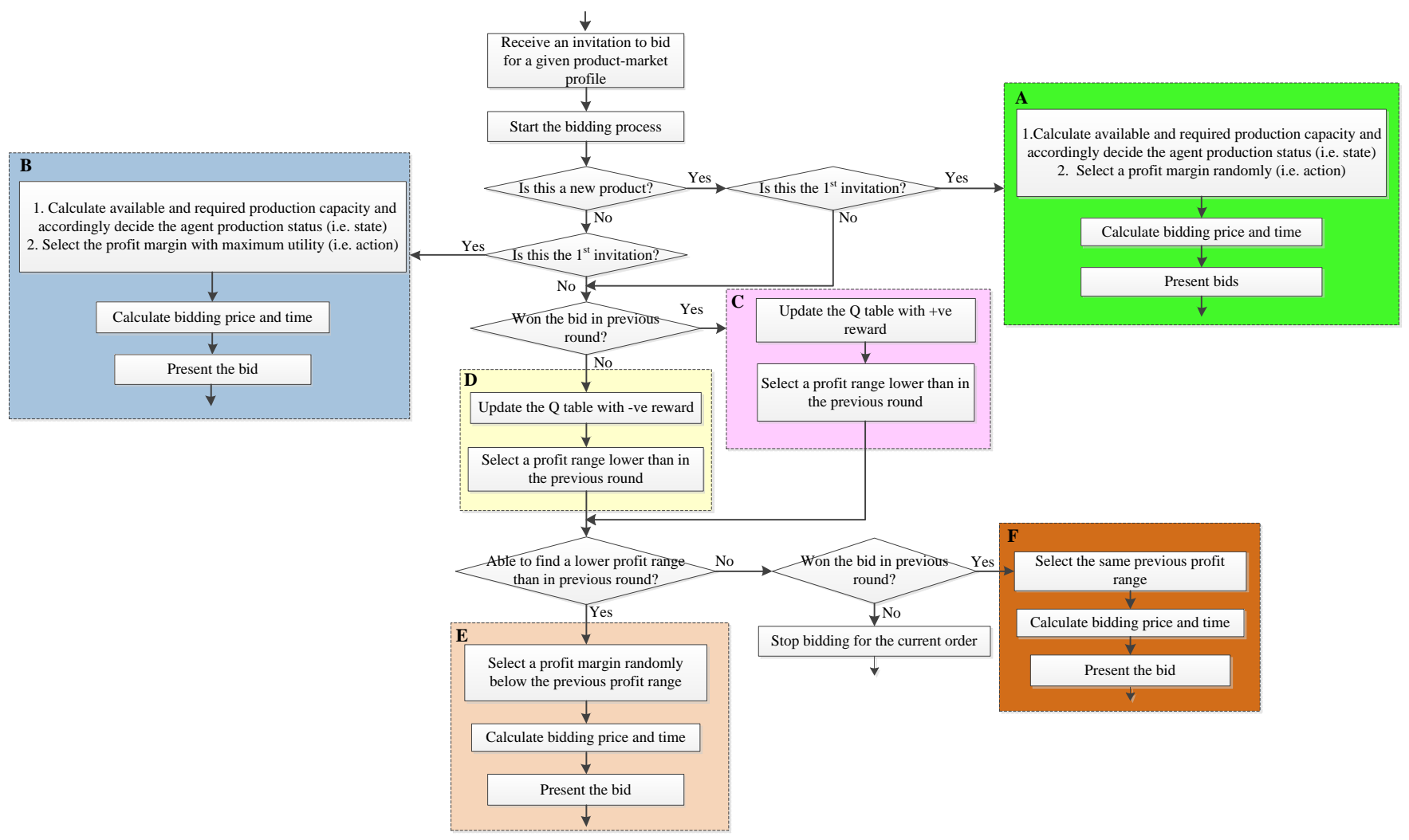

Fig. 2. Reasoning process of the physical agent 
invitation. Then the auction continues until the termination criteria is met (i.e. pre-defined number of iterations or at the time when there are no more capable physical agents to bid). The new invitation is generated by lowering the initial reserved values by a percentage value.

SES agents: suppliers/manufacturing facility/distribution centre selection agents are considered as the set of SES agents. The primary task of SES agents is to select feasible physical agents (candidate $R_{i j k}$ ) by comparing $R P_{i j k}$ with $B P_{i j k}$, and $R T_{i j k}$ with $B T_{i j k}$ as given in (19) and (20) respectively.

OPT agent: optimizes SN level performance in terms of total supply network cost (TSNC) and lead-time (LT) as given in (21) and (22) by optimally configuring the $\mathrm{SN}$ to suit a given product-market profile. The TSNC consists of the two primary elements, processing cost (PC) (i.e. the first term of (21)) and transportation cost (TC) (i.e. the second term of (21)). Similarly, LT includes processing time (PT) (i.e. the first term of (22)) and transportation time (TT) (i.e. the second term of (22)).

OPT agent receives candidates $R_{i j k}$ s from SES agents and then the optimal alternative SNCs are generated using NSGA-II subjected to the condition that one physical agent at each node for each stage is selected to cater the given product-market profile as per (23). The transportation cost for each SNC is obtained by contacting the TA agent.

From a mathematical point of view, generating optimal alternative SNCs belongs to the combinatorial optimization problem type, which cannot be solved with exhaustive search approaches in polynomial time. In this study we employed NSGA-II which is one of the popular evolutionary algorithms that has been used in a number of applications in many areas [35]. This algorithm initializes with a set of SNCs consisting of a physical agent from each node. Multiple objectives as in (21) and (22) of this problem are considered as fitness functions of

Minimize the TSNC:

$$
\begin{aligned}
= & \sum_{l \in L} \sum_{S_{i} \in S} \sum_{N_{i j} \in S_{i}} \sum_{R_{i j k} \in N_{i j}} y_{i j k} \cdot B P_{i j k} \cdot \delta_{i j} \cdot V_{l} \\
& +\sum_{S_{i} \in S} \sum_{N_{i j} \in S_{i}} \sum_{R_{i j k} \in N_{i j}} \sum_{S_{i^{\prime}} \in S} \sum_{N_{i^{\prime} j^{\prime} \in S_{i}}} \sum_{R_{i^{\prime} j^{\prime} k^{\prime} \in} \in N_{i^{\prime} j^{\prime}}} x_{i j k \rightarrow i^{\prime} j^{\prime} k^{\prime}} \cdot \alpha \cdot D_{i j k \rightarrow i^{\prime} j^{\prime} k^{\prime}}
\end{aligned}
$$

Minimize LT:

$$
\begin{aligned}
& =\sum_{l \in L} \sum_{S_{i} \in S} \sum_{N_{i j} \in S_{i}} \sum_{R_{i j k} \in N_{i j}} y_{i j k} \cdot B T_{i j k} \cdot \delta_{i j} \cdot V_{l} \\
& \quad+\sum_{S_{i} \in S} \sum_{N_{i j} \in S_{i}} \sum_{R_{i j k} \in N_{i j}} \sum_{S_{i^{\prime}} \in S} \sum_{N_{i^{\prime} j^{\prime}} \in S_{i} R_{i^{\prime} j^{\prime} k^{\prime}} \in N_{i^{\prime} j^{\prime}}} x_{i j k \rightarrow i^{\prime} j^{\prime} k^{\prime}} \cdot D_{i j k \rightarrow i^{\prime} j^{\prime} k^{\prime}} / V \\
& \sum_{k \in K_{j}} y_{i j k}=1 \\
& T T_{i j k \rightarrow i^{\prime} j^{\prime} k^{\prime}}=\left(D_{i j k \rightarrow i^{\prime} j^{\prime} k^{\prime}} \cdot x_{i j k \rightarrow i^{\prime} j^{\prime} k^{\prime}}\right) / V \\
& T C_{i j k \rightarrow i^{\prime} j^{\prime} k^{\prime}}=x_{i j k \rightarrow i^{\prime} j^{\prime} k^{\prime} \cdot} \cdot \alpha \cdot D_{i j k \rightarrow i^{\prime} j^{\prime} k^{\prime}} \\
& \mu=\mu+R C_{i j k t} /(1+\gamma)^{*} A C_{i j k} \\
& Q_{m n}=Q_{m n}+\mu_{1}\left(\delta_{i j} * V_{l} * P_{m n}\right)
\end{aligned}
$$

the NSGA-II. Based on those fitness functions, the initial population is ranked using the sorting algorithm known as Pareto-fast non-dominated (PF-ND) algorithm (see [35] for details). Genetic operators are performed on the initial solution namely: (i) selection, (ii) crossover, (iii) mutation and (iv) elitism to select the next generation. Elitism is the selecting of best performing supply entities for the next generation. The given genetic operators perform on the population on every iteration and that will continue until it meets the termination criteria generating the Pareto optimal front which contains alternative optimal SNCs.

TA agent: calculates the transportation cost and time as given in (24) and (25) between the physical agents in a given configuration. TA agent maintains a database with the information about the $\mathrm{D}_{\mathrm{kk}}, \alpha$ and $\mathrm{V}$.

EA agent: evaluates the optimal alternative SNCs based on other desired SN level performance measures (in addition to cost and time) and select one best SNC which suits to the given product-market profile. After a SNC is selected, all physical agents are informed through relevant SES agents to update their $O C_{i j k}$ as given in (26) and the $\mathrm{Q}$ tables with positive rewards as given in (27).

\section{SOLUTION APPROACH}

The overall arrangement of physical agents and auxiliary agents are shown in Fig. 3. Initially, the product-market profile of each consumer region is estimated in terms of $V_{l}, L T_{l}$ and $P_{l}$ and that information is passed on to the OP agent for processing product-market profile specifications to determine the relevant supply nodes and the number of units required from each supply node. The OP agent is connected to both the AU and SES agent and the OP agent sends indexes of supply nodes and other product-market profile attributes to AU agent. At the same time, indexes of supply nodes and number of units required from each node are sent to SES agent. When the SES agent receives information from the OP agent, all physical agents are announced with the required number of units. Physical agents make their own decisions (i.e. $B P_{i j k}$ and $B T_{i j k}$ ) based on their available resources and past experience. Those decisions are then communicated to the SES agent to compare the reserved values and the agent's bid upon which the feasible physical agents (i.e. $R_{i j k} \mathrm{~s}$ ) are selected. Until the AU agent stops generating reserved values upon meeting terminating criteria, after every iteration, all physical agents are informed of the outcome of their bids. At the end of the auctioning process, the

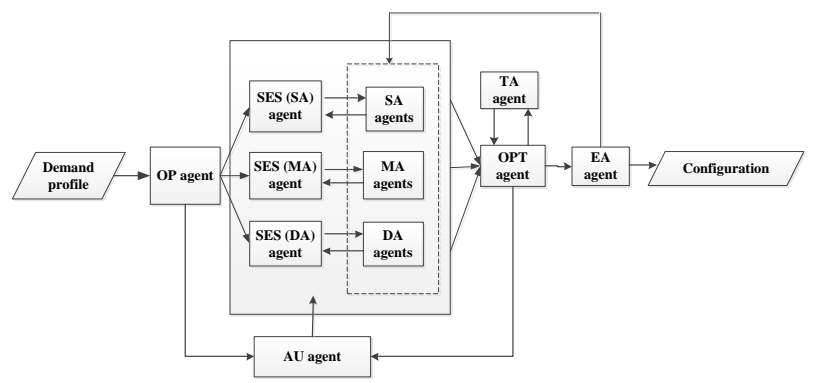

Fig. 3. Representation of the connectivity of agents 
candidate physical agents are sent to the OPT agent. The OPT agent contacts the TA agent on need basis to get the transportation cost and time. Once the OPT agent optimizes the SN in terms of TSNC and LT determining optimal alternative SNCs, EA agent is contacted to select the most suitable SNC based on the preference of the decision maker. In this study we consider sustainability as a key criterion. Once the best SNC is selected, then relevant physical agents are informed to update their $O C_{i j k}$ and their knowledge base. In any instance where the OPT agent is not able to find an optimal configuration for a given demand profile, then the productmarket profile is considered as baulked. In such cases the product-market profile attributes are re-evaluated to allow for feasible alternatives.

\section{CASE STUdy ANALYSIS}

We consider a dataset related to a refrigerator production network developed based on the resources drawn from [36-38], and introducing several other parameters as required to be tested using the proposed model.

This refrigerator SN consists of four stages; two supply stages (i.e. raw material, component/module), the final assembly stage and the distribution (of finished products) stage. There are four nodes in tier-2 supply stage, supplying raw material namely Iron, Plastic, Aluminium and Copper. Tier-1 supply stage provides the required 25 different components from five different supply nodes. In the manufacturing stage, the final products are assembled in order to dispatch to the distribution centers through which retailers at each consumer region receive goods. There are multiple entity options (i.e. $R_{i j k}$ ) available to perform the required value-adding functions at each node. We consider five consumer regions in Europe with derived product-market profiles considering the differences in price level index, population and individual income.

We carried out an experiment using a set of product-market profiles on the MAS model to generate alternative optimal
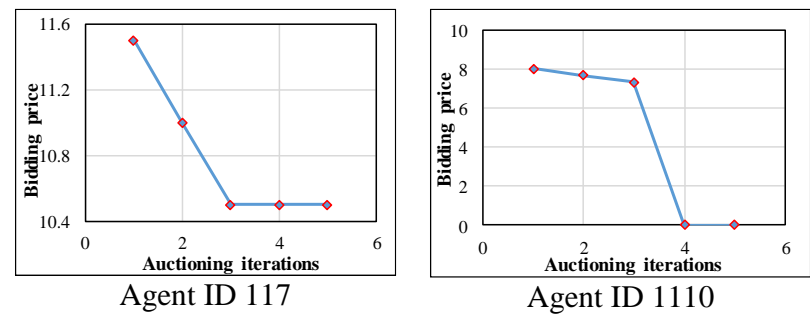

Fig. 4. Bidding price of agents for region 1 during the auctioning process
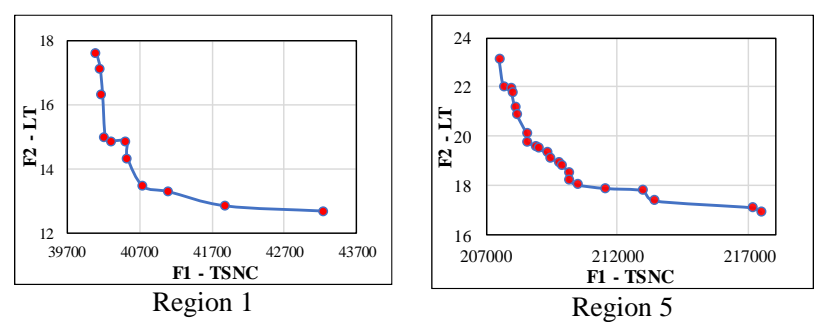

Fig. 5. Alternative optimal configurations for regional productmarket profiles generated by NSGA-II
TABLE III. ATtRibutes OF Alternative Optimal SNCS AND SUSTAINABLE SNC

\begin{tabular}{|c|l|c|c|c|c|c|}
\hline \multirow{2}{*}{$\begin{array}{c}\text { Demand } \\
\text { profile } \\
\left(\boldsymbol{Q}_{l}, \boldsymbol{D T}_{l,} \boldsymbol{P}_{l}\right)\end{array}$} & \multicolumn{2}{|c|}{ SNC solutions } & \multicolumn{2}{|c|}{ \% Saving } & \multicolumn{2}{|c|}{ Sustainable SNC } \\
\cline { 2 - 7 } & TSNC range & $\begin{array}{c}\text { LT } \\
\text { range }\end{array}$ & Cost & Time & $\begin{array}{c}\text { TSNC, \% } \\
\text { Saving }\end{array}$ & $\begin{array}{c}\text { LT, \% } \\
\text { Saving }\end{array}$ \\
\hline$(55,25,1100)$ & {$[729,786]$} & {$[13,18]$} & {$[34,29]$} & {$[48,28]$} & $(730,34)$ & $(17,32)$ \\
\hline$(45,22,1000)$ & {$[734,785]$} & {$[15,19]$} & {$[26,22]$} & {$[32,14]$} & $(762,24)$ & $(15,32)$ \\
\hline$(40,40,1350)$ & {$[788,858]$} & {$[26,32]$} & {$[37,42]$} & {$[35,20]$} & $(854,37)$ & $(26,35)$ \\
\hline$(120,35,1250)$ & {$[719,777]$} & {$[19,26]$} & {$[39,42]$} & {$[26,46]$} & $(772,38)$ & $(20,43)$ \\
\hline$(150,31,2000)$ & {$[1384,1449]$} & {$[17,24]$} & {$[28,31]$} & {$[23,45]$} & $(1447,28)$ & $(17,45)$ \\
\hline
\end{tabular}

SNCs which minimizes TSNC, as well as LT, while satisfying individual agent constraints and SN level constraints. Fig. 4 is an illustration of the outcomes (i.e. bidding values) of the auctioning process. The agent ID-117 kept reducing the bidding price for the first three iterations and then stayed at same price thereafter whereas agent ID-1110 quitted bidding after the third auction round. Fig. 5 shows the Pareto fronts generated by NSGA-II for the product-market profile of region 4 which indicates the TSNC and LT of alternative optimal SNCs.

On these optimal configurations, the sustainability aspect is measured in terms of energy consumption.The experiment results are presented in Table III, which includes TSNC and LT ranges for the Pareto-optimal SNCs and the performance (in terms of TSNC and LT) of the most sustainable SC. Simulation results show that the selected SNCs are capable of meeting environmental performance and cost targets while satisfying the respective product-market profiles.

\section{CONCLUSIONS}

The study reported in this paper adopted a multi-agent modelling approach implemented on the MATLAB 2016b platform along with auction-based agent interactions to model the individual decision-making behaviour of supply entities in a SN. Two types of agents (physical and auxiliary agent) were employed with distinct agent architectures to serve the intended purposes of the SNC problem. Simulation results generated through the application of this approach to a refrigerator $\mathrm{SN}$ demonstrated the adaptive behaviour of physical agents in terms of making competitive bids based on their resources availability. The alternative optimal SNCs generated using this competitive bidding process were evaluated against a set of SN conditions, sustainable goals (in terms of economic, business continuity and environmental performance) and the consumer specific requirements. The proposed model can assist decision makers with identifying the most sustainable SNs that meet a given set of product-market conditions, as well as evaluating the alignment between sustainable goals and product-market profiles at regular intervals. With minimal modifications, this model could also be used as a tool for supporting a number of other decisions such as developing contingency plans in case an established SNC is not able to operate as intended (due to unforeseen circumstances such as disruptions caused by internal or external events), finding the most common SNCs or competitive supply entities for a range of product-market profiles. From a practical point of view, the above considerations may help managers in initiating and maintaining suitable relationships with preferred supply chain partners, as well as assessing the capacity of the existing $\mathrm{SN}$ in terms of 
adapting to changing product-market profiles. As such, in future studies, the proposed model can be extended to account for factors such as reliability of supply entities and evaluating the compatibility among the selected set of supply entities, considering the overall SN level goals. Furthermore, there is room for testing the capacity of a given $\mathrm{SN}$ to achieve sustainable goals in light of other conditions such as disruptions and variations in product-market profiles.

\section{REFERENCES}

[1] A. Rajeev, R. K. Pati, S. S. Padhi, and K. Govindan, "Evolution of sustainability in supply chain management: A literature review," Journal of Cleaner Production, vol. 162, pp. 299-314, 2017.

[2] P. Ahi and C. Searcy, "A comparative literature analysis of definitions for green and sustainable supply chain management," Journal of Cleaner Production, vol. 52, pp. 329-341, 2013.

[3] R. Dubey, A. Gunasekaran, T. Papadopoulos, S. J. Childe, K. T. Shibin, and S. F. Wamba, "Sustainable supply chain management: framework and further research directions," Journal of Cleaner Production, vol. 142, pp. 1119-1130, 2017.

[4] H. Reefke and D. Sundaram, "Key themes and research opportunities in sustainable supply chain management - identification and evaluation," Omega, vol. 66, pp. 195-211, 2017.

[5] C. J. Corbett and R. D. Klassen, "Extending the horizons: environmental excellence as key to improving operations," Manufacturing \& Service Operations Management, vol. 8, no. 1, pp. 5-22, 2006.

[6] A. Jaeger, K. Matyas, and W. Sihn, "Development of an Assessment Framework for Operations Excellence (OsE), based on the Paradigm Change in Operational Excellence (OE)," Procedia CIRP, vol. 17, pp. 487-492, 2014.

[7] S. J. Rusev and K. Salonitis, "Operational Excellence Assessment Framework for Manufacturing Companies," Procedia CIRP, vol. 55, pp. 272-277, 2016.

[8] R. Edgeman and J. Eskildsen, "Modeling and Assessing Sustainable Enterprise Excellence," Business Strategy and the Environment, vol. 23, no. 3, pp. 173-187, 2014.

[9] J. A. Garza-Reyes, V. Kumar, S. Chaikittisilp, and K. H. Tan, "The effect of lean methods and tools on the environmental performance of manufacturing organisations," International Journal of Production Economics, vol. 200, pp. 170-180, 2018.

[10] M.A. Moktadir, T. Rahman, M.H. Rahman, S.M. Ali, and S.K. Paul, “ Drivers to sustainable manufacturing practices and circular economy: A perspective of leather industries in Bangladesh," Journal of Cleaner Production, vol. 174, pp.1366-1380, 2018.

[11] M. Eskandarpour, P. Dejax, J. Miemczyk, and O. Péton, "Sustainable supply chain network design: an optimization-oriented review," Omega, vol. 54, pp.11-32, 2015.

[12] A. Jaeger and K. Matyas, "Transformation of the EFQM approach from business towards operations excellence," Production Engineering, vol. 10, no. 3, pp. 277-291, 2016.

[13] R. Dubey, A. Gunasekaran, T. Papadopoulos, S. J. Childe, K. T. Shibin, and S. F. Wamba, "Sustainable supply chain management: framework and further research directions," Journal of Cleaner Production, vol. 142, pp. 1119-1130, 2017.

[14] W. Klibi, A. Martel, and A. Guitouni, "The design of robust valuecreating supply chain networks: a critical review," European Journal of Operational Research, vol. 203, no. 2, pp.283-293, 2010.

[15] M. J. Meixell and V. B. Gargeya, "Global supply chain design: A literature review and critique," Transportation Research Part E: Logistics and Transportation Review, vol. 41, no. 6, pp. 531-550, 2005.

[16] D. Mourtzis and M. Doukas, "Decentralized manufacturing systems review: challenges and outlook," Logistics Research, vol. 5, no. 3-4, pp. 113-121, 2012.

[17] R. Z. Farahani, S. Rezapour, T. Drezner, and S. Fallah, "Competitive supply chain network design: An overview of classifications, models, solution techniques and applications," Omega (United Kingdom), vol. 45, pp. 92-118, 2014.

[18] M. T. Melo, S. Nickel, and F. Saldanha-da-Gama, "Facility location and supply chain management - A review," European Journal of Operational Research, vol. 196, no. 2, pp. 401-412, 2009.

[19] R.Z. Farahani, S. Rezapour, T. Drezner, and S. Fallah, "Competitive supply chain network design: An overview of classifications, models, solution techniques and applications," Omega, vol. 45, pp.92-118, 2014.

[20] Y. F. Zhang, G. Zhang, T. Qu, Y. Liu, and R. Y. Zhong, "Analytical target cascading for optimal configuration of cloud manufacturing services," Journal of Cleaner Production, vol. 151, pp. 330-343, 2017.

[21] N. Shukla and S. Kiridena, "A fuzzy rough sets-based multi-agent analytics framework for dynamic supply chain configuration," International Journal of Production Research, vol. 54, no. 23, pp. 69846996, 2016

[22] E. Koberg and A. Longoni, "A systematic review of sustainable supply chain management in global supply chains," Journal of Cleaner Production, vol.207, pp.1084-1098, 2018.

[23] F. Jia, Y. Gong, and S. Brown,“ Multi-tier sustainable supply chain management: The role of supply chain leadership," International Journal of Production Economics, 2018.

[24] S. A. Melnyk, R. Narasimhan, and H. A. DeCampos, "Supply chain design: issues, challenges, frameworks and solutions," International Journal of Production Research, vol. 52, no. 7, pp. 1887-1896, 2013.

[25] P. Pourhejazy and O. K. Kwon, "The new generation of operations research methods in supply chain optimization: A review," Sustainability, vol. 8, no. 10, 2016.

[26] M. Wooldridge and N. R. Jennings, "Intelligent agents: Theory and practice," The knowledge engineering review, vol. 10, pp.115-152, 1995.

[27] S. Franklin and A. Graesser, "Is it an Agent, or just a Program?: A Taxonomy for Autonomous Agents," in International Workshop on Agent Theories, Architectures, and Languages, pp. 21-35, 1996.

[28] C. M. Macal and M.J. North, "Tutorial on agent-based modelling and simulation," Journal of simulation, vol. 4, pp.151-162, 2010.

[29] M. Wooldridge and N.R. Jennings, "Agent theories, architectures, and languages: a survey," International Workshop on Agent Theories, Architectures, and Languages, pp.1-39, 1994.

[30] K. O. Chin, K.S. Gan, R. Alfred, P. Anthony and D. Lukose, "Agent Architecture: An Overviews," Transactions on science and technology, vol. 1, pp.18-35, 2014.

[31] P. Maes, 'The agent network architecture (ANA)', SIGART bulletin, vol. 2, no. 4, pp. 115-120, 1991.

[32] M. V. Otterlo, "Markov Decision Processes: Concepts and Algorithms," Course on Learning and Reasoning, 2009.

[33] M. L. Puterman, 'Markov decision processes', Handbooks in operations research and management science, vol. 2, pp. 331-434, 1990.

[34] R. S. Sutton and A. G. Barto, "Reinforcement learning: An introduction," MIT press, 1998.

[35] K. Deb, K., A. Pratap, A., S. Agarwal, and T.A.M.T. Meyarivan, "A fast and elitist multiobjective genetic algorithm: NSGA-II," IEEE transactions on evolutionary computation, vol.6, no.2, pp.182-197, 2002.

[36] Y. Umeda, A. Nonomura, and T. Tomiyama, "Study on life-cycle design for the post mass production paradigm", AI EDAM, vol.14, pp.149-161, 2000 .

[37] H. R. Krikke, J. M. Bloemhof-Ruwaard, and I. N. Van Wassenhove, "Dataset of the refrigerator case: design of closed loop supply chains", 2001.

[38] M. Fleischmann, J.M. Bloemhof-Ruwaard, R. Dekker, E. Van Der Laan, J.A. Van Nunen, and L.N. Van Wassenhove, "Quantitative models for reverse logistics: A review," European Journal of Operational Research vol.103 (1), pp. 1-17,1997. 